\title{
Is the Reluctance for the Implantation of Right Kidneys Justified?: Reply
}

\author{
Denise M. D. Özdemir-van Brunschot ${ }^{1} \cdot$ Michiel C. Warlé
}

Published online: 5 May 2017

(C) Société Internationale de Chirurgie 2017

\section{Dear Editor,}

We agree with Khan et al. that there are several technical approaches for a successful implantation of a right living donor kidney with a short renal vein. As very well illustrated by two examples, Khan et al. state that procuring a renal vein with a small caval rim during open donor nephrectomy may be helpful to facilitate the venous anastomosis. This raises the question whether an open approach for donor nephrectomy should be advocated for the procurement of right living donor kidneys, especially when a short renal vein is expected. At this stage we feel that it is not justified to expose living donors to a more invasive open technique in centers where the laparoscopic technique is available.

In our view there are alternative strategies to overcome the problem of a right living donor kidney with a short renal vein. The first strategy is to accept the left kidney despite its greater size and/or the presence of multiple renal arteries. In our center the feasibility of this strategy is reflected by a relatively high percentage of left-sided living donor nephrectomies (approximately 85\%). Since we adopted this strategy, our technical failure rate has been below average. Once a right living donor kidney with a short renal vein has been procured, there are two technical approaches to achieve a tension-free venous anastomosis. The first approach is to implant a right kidney with a short renal vein into the recipients' right iliac fossa to allow an anastomosis of the renal vein to the recipients' caval vein that is situated more superficially (ventral) as compared to the iliac veins. Therefore, less vein length is

Denise M. D. Özdemir-van Brunschot od.od.ozdemir@gmail.com

1 Department of Vascular Surgery, Augusta Krankenhaus, Amalienstraße 9, 40472 Düsseldorf, Germany

2 Department of Vascular and Transplant Surgery, Radboud University Medical Center, Nijmegen, The Netherlands required for a tension-free anastomosis. Disadvantages of this approach are the need for a longer incision and a more difficult arterial anastomosis since implantation of a right kidney in the right iliac fossa means that the donors' artery and vein have to cross. This may increase the risk of kinking and/or compression of the renal vein. Nevertheless, in our center we usually implant a right kidney with a short renal vein into the recipients' right iliac fossa with good results. Another, more theoretical approach to overcome the problem of a short renal vein is to divide the recipients' internal iliac vein. This mobilizes both the common and external iliac vein enabling a more superficial (ventral) position. This also helps to perform a tension-free venous anastomosis. Nevertheless, there may be an increased risk of a major venous bleeding, and therefore, we feel this should be considered as a last resort solution.

In the Netherlands almost 30\% of living donor kidneys are right kidneys and $48.5 \%$ of deceased donor kidney transplantations are performed with a right kidney [1]. The latter indicates that right kidneys of deceased donors tend to be discarded somewhat more often as compared to their left counterparts. Apparently transplant surgeons are more reluctant to implant right kidneys. Given the higher technical failure rate, we feel that this reluctance is justified [1]. In our view the presence of a short renal vein should not be a reason to discard deceased donor kidneys or to perform an open procedure to procure a right living donor kidney. In centers where the laparoscopic technique for living donor nephrectomy is not available, the procurement of a renal vein with a small caval rim could be an alternative strategy to deal with a short renal vein.

\section{References}

1. Ozdemir-van Brunschot DMD, van Laarhoven CJHM, van der Jagt MFP et al (2016) Is the reluctance for the implantation of right donor kidneys justified? World J Surg 40:471-478. doi:10.1007/ s00268-015-3232-0 